\section{Referências bibliográficas}

1. Scheinhorn DJ. Stearn-Hassenpflig, M. Provision of long-term mechanical ventilation. Crit Care Med 1998; 14: 819-32.

2. Cohen IL, Booth FVM. Cost containment and mechanical ventilation in the United States. New Horizons 1994; 2: 283.

3. Goldberg AI. Outcomes of home care for life-supported persons: long term oxygen and prolonged mechanical ventilation [Editorial]. Chest 1996; 109: 595.

4. Chailleux E, Fauroux B, Binet F et al. Predictors of survival in patients receiving domiciliar oxygen therapy of mechanical ventilation: a 10 year analysis of ANTADIR observatory. Chest 1996; 109: 741.

5. Venkataraman ST. Noninvasive mechanical ventilation and respiratory care. New Horizons 1999; 7: 353-8.
6. Padman R, Lawless S, Von Nessen S. Use of BiPAP by nasal mask in the treatment of respiratory insufficiency in pediatric patients: preliminary investigation. Pediatr Pulmonol 1994; 17: 119-23.

7. Fortenberry JD, Del Toro J, Jefferson LS et al. Management of pediatric acute hypoxemic respiratory insufficiency with bilevel positive (BiPAP) nasal mask ventilation. Chest 1995: 108: 1059-64.

8. Padman R, Lawless ST, Kettrick RG. Noninvasive ventilation via bilateral positive airway pressure support in pediatric practice. Crit Care Med 1998; 26: 169-73.

9. Pilbeam SP. Home mechanical ventilation. In: Pilbeam SP. Mechanical ventilation. Physiological and clinical applications. $3^{\text {rd }}$ ed. St Louis: Mosby; 1998, p.385-412.

\title{
Refluxo gastroesofágico e doença respiratória na infância
}

\author{
Gastroesophageal reflux and respiratory diseases in children
}

\author{
José Dirceu Ribeiro*
}

\begin{abstract}
Acredita-se que a primeira observação de que desordens respiratórias pudessem estar sob influência do trato digestivo data de 1776, quando Nicholas Rosen von Rosenstein, no seu livro The Diseases of Children and their Remedies, descreveu o que ele chamou de tosse gástrica da infância. Em seguida, William Heberden, em 1802, sugeriu que, em asmáticos, “a respiração é mais curta e mais difícil após uma refeição", e Sir William Osler, em 1892, em seu livro The Principles and Practice of Medicine, relatou que "paroxismos graves de asma podem ser induzidos quando o estômago é sobrecarregado, ou quando usados determinados tipos de alimentos"1.

Entretanto, foi em 1946 que Mendelson provou, em seu estudo clássico sobre aspiração pulmonar durante o ato anestésico, que sintomas sugestivos de obstrução brônquica podem ser causados por aspiração do conteúdo gástrico. Em 1949, pneumonias recidivantes foram observadas por Belcher como complicação de processos esofagianos obstrutivos, levando o nome de "pneumonite por disfagia".
\end{abstract}

\footnotetext{
* Mestre em Clínica Médica, Doutor em Pediatria e Professor de Pediatria da FCM-Unicamp. Vice-presidente do Departamento de Pneumologia da SBP.
}

Finalmente, em 1962, Kennedy abriu uma nova era sugerindo que o refluxo gastroesofágico (RGE) "silencioso ou oculto" pode ser uma causa importante e pouco reconhecida de enfermidades pulmonares. A partir dessa época numerosos estudos clínicos mostraram associação da doença do RGE (DRGE) e uma variedade de sinais, sintomas e doenças do aparelho respiratório ${ }^{1-3}$.

O RGE pode ocorrer de maneira fisiológica em qualquer indivíduo. O termo doença do refluxo gastroesofágico tem sido utilizado para descrever o amplo espectro de distúrbios causados pelo RGE. A distinção entre RGE fisiológico e DRGE é feita em função da freqüência e intensidade do $\mathrm{RGE}^{4}$. No entanto, apesar da associação entre doenças respiratórias e RGE ser bem reconhecida, o significado exato desta relação e suas implicações clínicas ainda permanecem controversos.

A DRGEé uma doença multifatorial, na qual os eventos iniciais ainda são desconhecidos e uma prevalência aumentada de RGE em pneumopatas não indica por si só uma relação causal.

Se em indivíduos saudáveis temos o RGE fisiológico normal, no outro extremo temos indivíduos com esofagite grave. Entre os dois extremos comprovam-se vários graus (intensidade) e tipos (causas) de RGE (funcional, patológico, secundário). 
O momento do início da doença respiratória associada ao RGE permanece desconhecido. A comunidade científica está tentando encontrar a resposta para esse momento. Nesse sentido, o trabalho de Meyer e Fischer publicado nesta edição do Jornal de Pediatria vem preencher uma lacuna importante.

As contradições existentes na literatura, sobre a interação RGE e doença respiratória, podem ser explicadas pelo simples fato de que há três grupos de pacientes:

I - pacientes com doença respiratória e RGE não relacionados entre si;

II- pacientes com doença respiratória favorecendo RGE;

III- pacientes com RGE favorecendo a doença respiratória, através dos seguintes mecanismos: ${ }^{1,5-8}$

RGE causando doença respiratória por: micro ou macroaspiração de conteúdo gástrico; reflexo vagal; aumento da hiperreatividade brônquica; liberação de taquicininas.

Doença respiratória causando RGE por: aumento do gradiente de pressão transdiafragmática; retificação do diafragma pela hiperinsuflação crônica; uso de medicamentos (metilxantinas/ simpaticomiméticos, antibióticos) para tratar a doença respiratória e tosse induzindo RGE.

Embora a pHmetria esofágica de 24 horas seja reconhecida como o "padrão ouro" para o diagnóstico de RGE, ${ }^{9}$ esse método detecta apenas os refluxos ácidos $(\mathrm{pH}<4)$ e os alcalinos $(\mathrm{pH}>7)$. Os RGE com $\mathrm{pH}$ entre 5-6,8, podem representar muitos episódios de RGE não documentados pela $\mathrm{pHmetria.}$

Wenzl e col. ${ }^{10}$, em 1999, estudaram RGE em crianças com doença respiratória, utilizando, concomitantemente, os métodos de pHmetria e impedância intraluminal (IMP). Verificaram, em 22 crianças, 364 episódios de RGE detectados por IMP, mas apenas $11,4 \%$ tiveram $\mathrm{pH}<4$, também documentados pela pHmetria. Dentre os 364 episódios de RGE, 312 estavam associados com anormalidades respiratórias, mas somente $11,9 \%$ tinham $\mathrm{pH}<4$ na pHmetria. De 19 episódios de queda da saturação de oxigênio maior que $10 \%$ do valor inicial, apenas $3(15,8 \%)$ apresentaram valores de $\mathrm{pH}<4$ na pHmetria. Nesse estudo, 77,6\% dos episódios de RGE, acompanhados de apnéia, foram documentados apenas pela IMP e não foram documentados pela pHmetria. Os autores concluíram que apenas o uso da pHmetria de 24 horas não detecta a maioria dos episódios de RGE associados a sintomas respiratórios. A técnica de IMP, que detecta RGE independente do $\mathrm{pH}$ do conteúdo refluído (principalmente aqueles com pH entre 5 e 6,8), promete ser uma ferramenta fundamental para estudos que tentam correlacionar RGE e sintomas respiratórios.

Apesar das dúvidas, controvérsias e dificuldades descritas, muitos autores, nas últimas duas décadas, têm tentado esclarecer os mecanismos envolvidos na relação das diversas modalidades de doenças respiratórias e RGE. O estudo de Meyer \& Fischer e o de Wenzl e col. ${ }^{10}$ atendem uma necessidade clínica, ao propor, de uma maneira pioneira, um fenômeno dentro da interação RGE e doença respiratória, que pode ser observado com a queda nos valores de saturação transcutânea do oxigênio. Abrem-se, com esses trabalhos, mais oportunidades de pesquisa que poderão permitir melhor entendimento da relação, ainda confusa e não completamente conhecida, entre RGE e doença respiratória. Com certeza esses trabalhos serão referências para outros estudos futuros.

\section{Referências bibliográficas}

1. De Campos-Mello AL. Avaliação do refluxo gastroesofágico em crianças e adolescentes asmáticos atópicos graves com e sem pneumonias recidivantes [dissertação de mestrado]. Campinas, SP: Curso de Pós-graduação em Pediatria da FCM Unicamp; 1999. p.80.

2. Quintela TMM. Avaliação de refluxo gastroesofágico na morbidade de lactentes chiadores atópicos e não atópicos [tese de doutorado]. Campinas, SP: Curso de Pós-graduação em Medicina Interna da FCM Unicamp; 1997. p.183.

3. Kennedy JH. "Silent gastroesophageal reflux": an important but little known cause of pulmonary complications. Dis Chest 1962; 42: 42-5.

4. Vandenplas Y, Ashkenasi A, Belli D, Boige N, Bouquet J, Cadranel S, et al. A proposition for the diagnosis and treatment of gastroesophageal reflux disease in children: a report from a working group on gastroesophageal reflux disease. Eur J Pediatr 1993; 152:704-11.

5. Field SK, Evans JÁ, Price LM. A critical review of the studies of the effects of simulated or real gastroesophageal reflux on pulmonary function in asthmatic adults. Chest 1999; 115:848-56.

6. Lodi U, Harding, Scoghlan C, Guzzo MR, Walker LH. Autonomic regulation in the asthmatics with gastroesophageal reflux. Chest 1997; 11:65-70.

7. Eid NS, Shephard RW, Thomson MA. Persistent wheezing and gastroesophageal reflux in infants. Pediatric Pulmonology 1994; 18:39-44.

8. Vandenplas Y. Asthma and gastroesophageal reflux. J Pediatr Gastroenterol Nutr 1997; 24: 89-99.

9. Mezzacapa MA, Collares EF. Utilização da monitorização prolongada do pH esofágico no diagnóstico da doença do RGE em recém nascidos. J Pediatr (Rio J) 1999; 75: 237-43.

10. Wenzl TG, Silny J, Schenke S, Peschgens T, Heimann G, Skopnik H. Gastroesophageal reflux and respiratory phenomena in infants: status of the intraluminal impedance technique. $\mathbf{J}$ Pediatr Gastroenterol Nutr 1999;28:423-8.

Endereço para correspondência:

Dr. José Dirceu Ribeiro

Centro de Investigação em Pediatria

Depto. Pediatria - Fac. de Ciências Médicas da Unicamp

Campinas - SP - Caixa Postal 6111 - CEP 13083-970

E-mail: dirceu@head.fcm.unicamp.br

Fone: (19) 3289.3874 / Fax: (19) 3788.8970 